\title{
Size Dependent Hardness of Polyamide/Imide
}

\author{
Ramajaneyulu V.S. Tatiraju ${ }^{1}$, Chung-Souk Han ${ }^{*}, 1$ and Svetoslav Nikolov ${ }^{2}$ \\ ${ }^{I}$ North Dakota State University, Department of Civil Engineering, Fargo, ND 58105, USA \\ ${ }^{2}$ Max-Planck-Institut für Eisenforschung, Max-Planck-Str. 1, 40237 Düsseldorf, Germany
}

\begin{abstract}
Indentation experiments with indentation depths in the nano- and micrometer ranges in the literature have shown that that an indentation size effect similar to metals is present in polymers. In this paper we present indentation experiments of a polyamide/imide polymer of indentation depths in the range of 1 to 30 microns. An increase in hardness of about $70 \%$ was observed at small indentation depths compared to hardness at large indentation depths. The indentation results are discussed with respect to an indentation hardness rule based on Frank elasticity suggested in a previous paper by the authors. It is found that the hardness model agrees well with the experiments.
\end{abstract}

\section{INTRODUCTION}

The indentation size effect manifests itself in increasing hardness with decreasing indentation depth and is fairly well investigated for metals. For polymers these effects are not well understood and investigated. The understanding of the size dependent deformation behavior is yet not only of importance for small dimensioned components in MEMS/ NEMS applications but also for the development of advanced composite materials with small material phases. In Han \& Nikolov 2007 [1] indentation experiments in the literature have been summarized and analyzed and it has been found that the indentation size effect strongly depends on the polymeric material. For instance, ultra high molecular weight polyethylene (UHMWPE) [2] and polytetraflourethylene/Teflon (PTFE) [3] do not seem to show any indentation size effects, while epoxy and polycarbonate show significant indentation size effects at indentation depths of $100 \mathrm{~nm}$ up to several microns [4].

Here commercially obtained polyamide/polyimide sheets known also under the brand names Kerimid and Torlon are investigated which have very high strength for an unreinforced thermoplastic. The considered polyamide/polyimide sheets contain aromatic rings in their molecular structure and have - according to the sample fabricator - very good high temperature properties, good wear and radiation resistance, inherently low flammability and smoke emission. Applications include parts for internal combustion and jet engines, bearings and thrust washers and mechanical, electrical and electronic components. In this paper the results of indentation size effect tests of polyamide/imide sheets are presented where significant hardness increases at indentation depths below 10 microns have been observed. These results are evaluated with the hardness model suggested by Han \& Nikolov [1] and it is found that the model and the experimental results are in good agreement. These findings are discussed with respect to other results on the size effects of polymers in the literature.

*Address correspondence to this author at the North Dakota State University, Department of Civil Engineering, Fargo, ND 58105, USA; Tel: +1 (701) 231-5647; E-mail: chung-souk.han@ndsu.edu

\section{INDENTATION EXPERIMENTS}

The polyamide/imide sheets with $6 \mathrm{~mm}$ thickness were obtained from the Goodfellow cooperation, Oakdale, PH, USA. The surface of these samples was very smooth so that it appeared that grinding or polishing would not have improved the quality of the surface. The general description of the sample fabricator states that the polyamide/imide sheet material is an amorphous, strongly colored, high performance polymer with linear predominantly aromatic molecules of high thermal stability.

The indentation tests were performed with a Fischerscope HM2000S indenter with a Berkovich tip (Fischer Technology, Inc., USA). The loading and unloading time of the load controlled indentations were chosen to be 20 seconds with zero seconds holding time to minimize creep type deformation. These loading conditions should result in the same strain rate distribution relative to the maximal indentation depth irrespective of the actual maximal load [2].

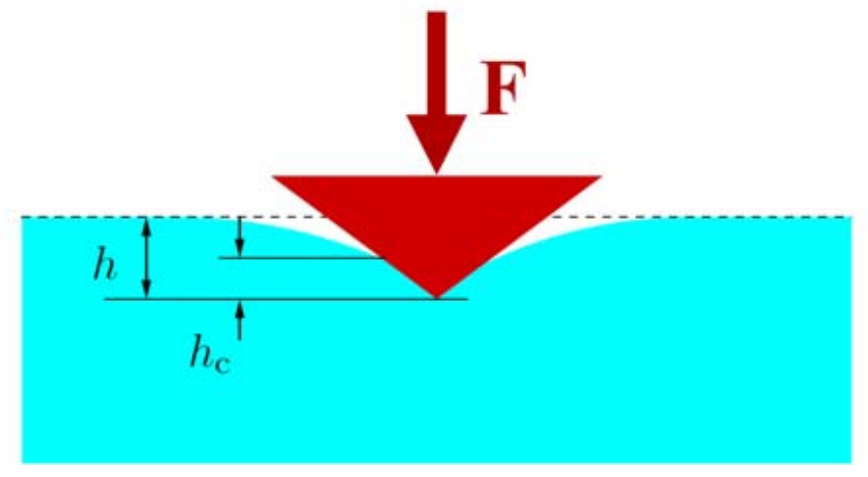

Fig. (1). Indentation depths $h$ and $h_{\mathrm{c}}$.

For clarity of the notation in Fig. (1) the indentation depth $h$ and its corrected (according to Oliver \& Pharr 1992 [5]) counterpart $h_{\mathrm{c}}$ are shown. In Fig. (2) the elastic - $W_{\mathrm{I}}^{\mathrm{e}}-$ and inelastic $-W_{\mathrm{I}}^{\mathrm{p}}$ - parts of the total indentation work

$W_{\mathrm{I}}=W_{\mathrm{I}}^{\mathrm{e}}+W_{\mathrm{I}}^{\mathrm{p}}$ 
in the loading and unloading sequence of the loaddisplacement curve are illustrated.

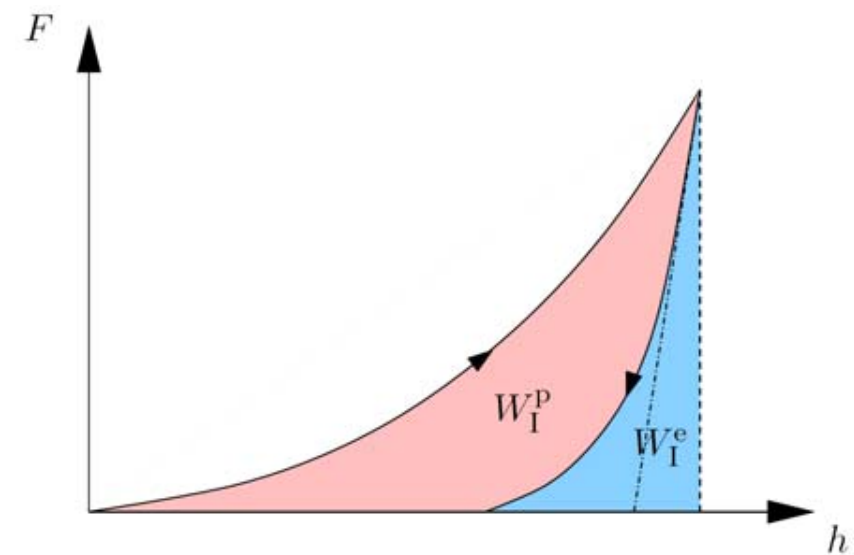

Fig. (2). Elastic and plastic parts of indentation work in a loaddisplacement diagram (bottom).

A typical load-displacement curve of the polyamide/imide sheet is shown in Fig. (3). As can be seen therein and from Fig. (4) where the ratio between $W_{\mathrm{I}}^{\mathrm{e}}$ and $W_{\mathrm{I}}$, i.e. $\eta=W_{\mathrm{I}}^{\mathrm{e}} / W_{\mathrm{I}}$ is shown for indents at different locations and loads the elastic and plastic parts of the indentation work are about the same with a slight tendency towards $W_{\mathrm{I}}^{\mathrm{p}}$ for decreasing $h$. Such a tendency is also observed in other polymers (see, e.g., [2]). The indentation size effect can be assessed by the hardness versus indentation depth relation shown in Fig. (5). Therein both the universal hardness $H_{\mathrm{u}}=F /\left(c_{\mathrm{Bu}} h^{2}\right)$ and the indentation hardness $H_{\mathrm{i}}=F /\left(c_{\mathrm{Bi}} h_{\mathrm{c}}^{2}\right)$ have been plotted, where $c_{\mathrm{Bu}}=26.43$ for the universal hardness and $c_{\mathrm{Bi}}=23.96$ is the geometric constant to relate $h_{\mathrm{c}}$ to the contact area $A_{\mathrm{c}}$ for the Berkovich indenter tip [6]. If no size effects would be present both hardness values should be independent of the indentation depth $h$. As can be seen in Fig. (5), however, significant increases in the hardness have been determined for the polyamide/imide sheets with about a $2 / 3$ increase in an indentation depth range of 1.5 to 30 microns for both $H_{\mathrm{i}}$ and $H_{\mathrm{u}}$.

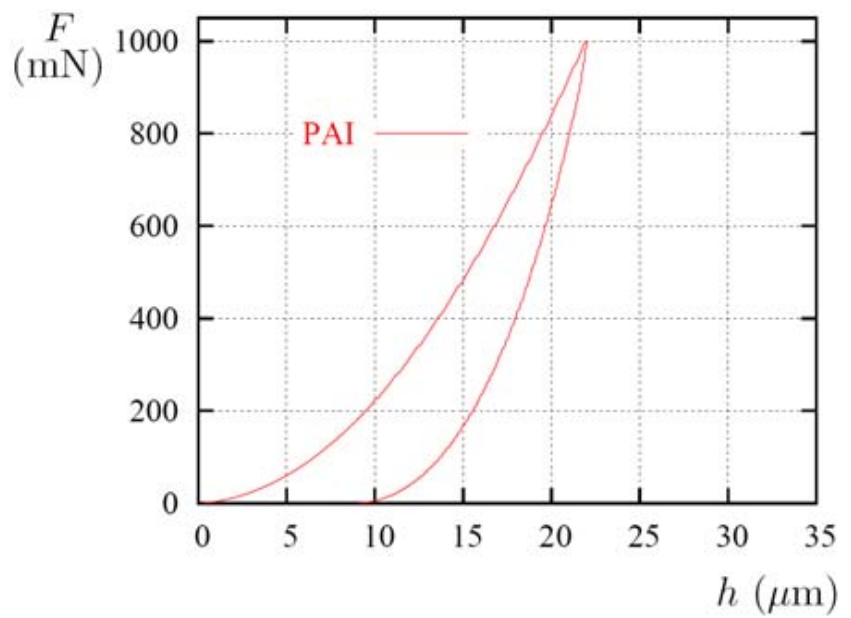

Fig. (3). Indentation depth $h$ versus applied load $F$.

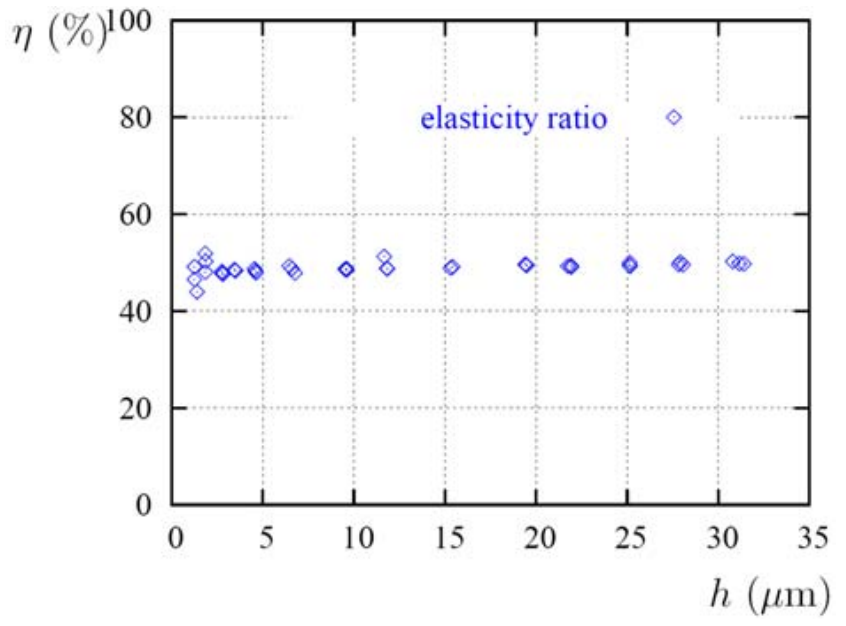

Fig. (4). Ratio $\eta$ versus indentation depth $h$.

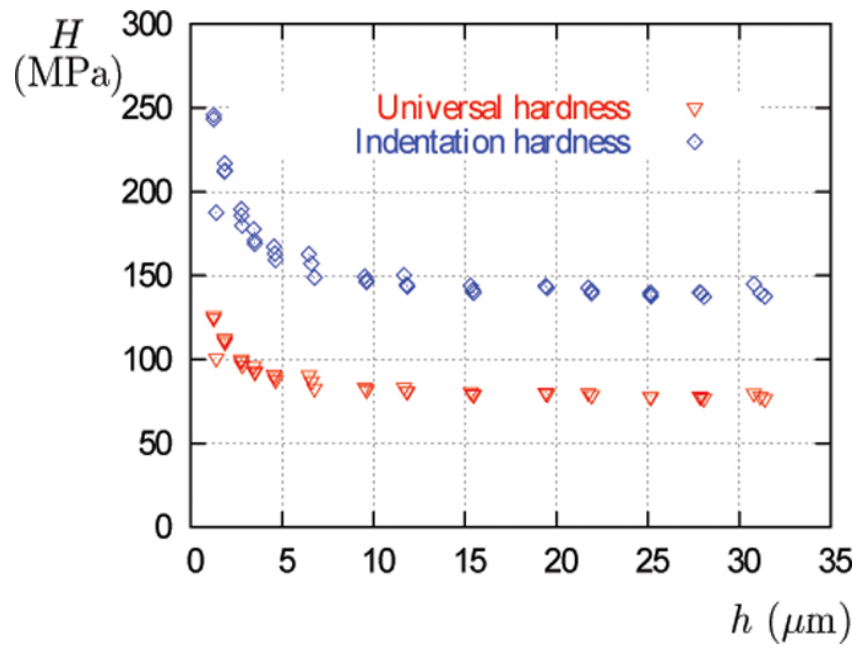

Fig. (5). Indentation $H_{\mathrm{i}}$ and universal hardness $H_{\mathrm{U}}$ versus indentation depth $h$.

\section{ANALYSIS AND DISCUSSION OF EXPERIMENTAL RESULTS}

Assuming that Frank energy [7] known to be important in liquid crystal polymers are also relevant in amorphous polymers a Frank elasticity type rotation gradient energy was suggested in Nikolov et al. [8] to account for observed elastic size effects in the bending of microbeams $[9,10]$. The Frank energy is a quadratic function in the gradients of the orientations of the more rigid (nematic) parts of the polymer chain and is also directly proportional to the Frank constant (in its one parameter form) $K$ which depends on the molecular structure and properties of the polymer (see, e.g., [11]). A corresponding extension for elasto-plastic materials was suggested and a related hardness model was deduced in Han \& Nikolov [1] by including a Frank energy related indentation work term $W_{\mathrm{I}}^{\mathrm{F}}$ into the total indentation work, i.e.

$W_{\mathrm{I}}=W_{\mathrm{I}}^{\mathrm{e}}+W_{\mathrm{I}}^{\mathrm{p}}+W_{\mathrm{I}}^{\mathrm{F}}$, 
where $W_{\mathrm{I}}^{\mathrm{F}}$ should become small relative to $W_{\mathrm{I}}^{\mathrm{e}}+W_{\mathrm{I}}^{\mathrm{p}}$ for large indentation depths. With decreasing indentation depths however the energy $W_{\mathrm{I}}^{\mathrm{F}}$ to be exerted during indentation will increase relative to $W_{\mathrm{I}}^{\mathrm{e}}+W_{\mathrm{I}}^{\mathrm{p}}$ resulting in higher applied forces and correspondingly higher hardnesses.

The hardness model deduced from (2) in [1]

$H=H_{0}\left(1+\frac{c_{\ell}}{h}\right)$,

is formulated with a length scale parameter $c_{\ell}$ and a macroscopic hardness $H_{0}$ which is also the lower limit of $H$. It is found that (3) describes the $h-H$ relation fairly well for epoxy, polycarbonate, and polystyrene for which experimental data of up to 10 microns were reported in the literature $[2,4]$. For small indentation depths of approximately below $100 \mathrm{~nm}$ surface roughness and surface effects may be present which the relation (3) does not take into account and consequently (3) does not represent the size dependent hardness below about $100 \mathrm{~nm}$ indentation depth. The relation (3) was also found to predict the $h-H$ relation for silicone rubber at much higher indentation depths of up to 100 microns [1]. As can be seen from Fig. (6) where $1 / h$ is used for the $x$-axis relation (3) also describes the hardness - indentation depth behavior of the investigated polyamide/imide sheets fairly well for both indentation and universal hardness with their linear fits with respect to $1 / h: H_{\mathrm{i}}=135.4 \mathrm{MPa}(1+$ $0.947 \mu \mathrm{m} / \mathrm{h})$ and $H_{\mathrm{u}}=76.65 \mathrm{MPa}(1+0.751 \mu \mathrm{m} / \mathrm{h})$.

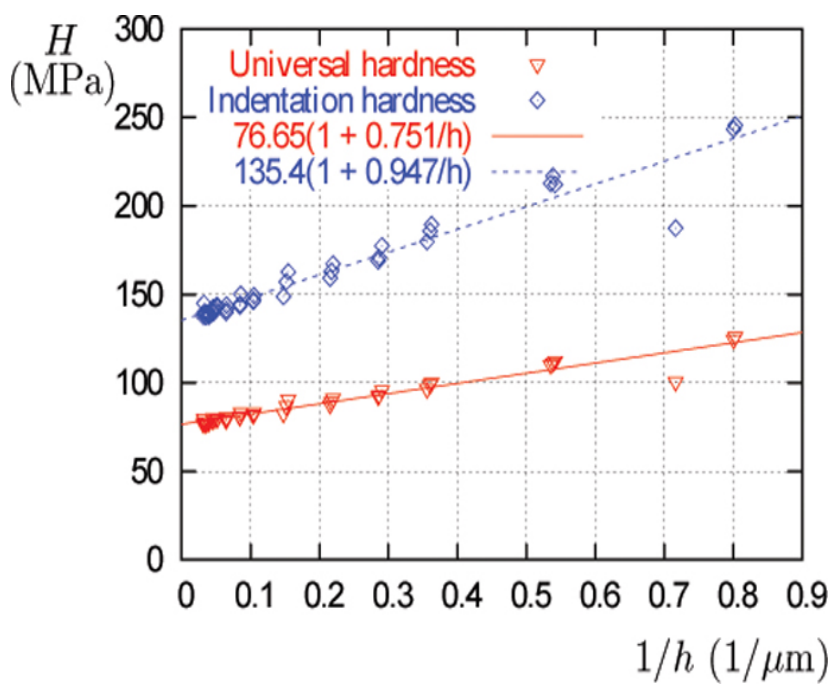

Fig. (6). Indentation $H_{\mathrm{i}}$ and universal hardness $H_{\mathrm{U}}$ versus inverse of indentation depth $1 / h$.

It is interesting to note that Nylon as an polyamide shows a significantly different characteristic in its indentation depth dependent hardness. In Shen et al. [12] Nylon 66 was indented in indentation depth by between about 150 and $5000 \mathrm{~nm}$. Below about 500nm the determined hardness increased with decreasing $h$. However, in their experiments the hardness did not decrease for $\mathrm{h}$ larger than about $600 \mathrm{~nm}$, i.e. a lower saturation limit was reached at about $h=600 \mathrm{~nm}$. For the investigated polyamide/imide samples such a saturation in the hardness has not been observed for the tested $h$-range of up to $30 \mu \mathrm{m}$. The differences in the depth dependent hardness characteristics may lie in the differences of the molecular structure of Nylon 66 and the polyamide/imide. As mentioned earlier the Frank energy constant $K$ depends on the molecular structure and properties of the polymer. The aromatic rings of the indented polyamide/imide will be result in higher bending rigidity compared to those of Nylon which is according to Liu \& Fredrickson [11] affects the Frank energy which may explain the differences between Nylon in Shen et al. [12] and the here investigated polyamide/imide material.

\section{CONCLUSIONS}

It may be worth noting that indentation size effects have also been observed in metallic glass [13] and therefore the indentation size effect can not be only be related to the notion of geometrically necessary dislocations. In the here investigated polymer polyamide/imide it is believed that the indentation size effect can be rationalized by the notion of the Frank energy which is increasing with increasing rotational gradients. The indentation tests presented here also show that the size effect in polymers can be of significance at indentation depths above one micron and that the hardness law $H=H_{0}\left(1+c_{\ell} / h\right)$ predicts the depth dependent hardness very well.

\section{ACKNOWLEDGMENTS}

The support of this work by the North Dakota EPSCoR Program (EPS-0447679) is highly appreciated.

\section{REFERENCES}

[1] Han CS, Nikolov S. Indentation size effects of polymers and related rotation gradients. J Mater Res 2007; 22: 1662-72.

[2] Briscoe BJ, Fiori L, Pelillo E. Nanoindentation of polymeric surfaces. J Phys D Appl Phys 1998; 31: 2395-405.

[3] Li X, Bhushan B. Continuous stiffness measurement and creep behavior of composite magnetic tapes. Thin Solid Films 2000; 377378: 401-6.

[4] Chong ACM, Lam DCC. Strain gradient plasticity effect in indentation hardness of polymers. J Mater Res 1999; 14: 4103-10.

[5] Oliver WC, Pharr GM. Improved technique for determining hardness and elastic modulus using load and displacement sensing indentation experiments. J Mater Res 1992; 7: 1564-80.

[6] ISO 14577-1. Metallic materials - Instrumented indentation test for hardness and materials parameters - Part 1: test method. International Organization for Standardization, Geneva, Switzerland, 2002.

[7] de Gennes PG, Prost J. The Physics of Liquid Crystals. Oxford University Press, 1993.

[8] Nikolov S, Han CS, Raabe D. On the origin and modeling of size effects in small-strain elasticity of solid polymers. Int J Sol Struct 2007; 44: 1582-1592. Corrigendum: Int J Sol Struct 2007; 44: 7713.

[9] Lam DCC, Yang F, Chong ACM, Wang J, Tong P. Experiments and theory in strain gradient elasticity. J Mech Phys Sol 2003; 51: 1477-508.

[10] McFarland AW, Colton JS. Role of material microstructure in plate stiffness with relevance to microcantilever sensors. J Micromech Microeng 2005; 15: 1060-67.

[11] Liu AJ, Fredrickson JH. Free energy functionals for semiflexible polymer solutions and blends. Macromolecules 1993; 26: 2817. 
[12] Shen L, Liu T, Lv P. Polishing effect on nanoindentation behavior of nylon 66 and its nanocomposites. Polymer Test 2005; 24:746749 .
[13] Sort J, Van Steenberge N, Gimazov A, et al. Study of the mechanical behaviour of a zr-based metallic glass rod using micro- and nano-indentation. Open Mater Sci J 2008; 2: 1-5.

Received: March 31, 2008

Revised: May 07, 2008

Accepted: August 07, 2008

(C) Tatiraju et al.; Licensee Bentham Open.

This is an open access article licensed under the terms of the Creative Commons Attribution Non-Commercial License (http://creativecommons.org/licenses/by-nc/3.0/) which permits unrestricted, non-commercial use, distribution and reproduction in any medium, provided the work is properly cited. 